\title{
Smooth Quantum Hydrodynamic Model Simulation of the Resonant Tunneling Diode
}

\author{
CARL L. GARDNER* and CHRISTIAN RINGHOFER ${ }^{\dagger}$ \\ Department of Mathematics, Arizona State University, Tempe, AZ 85287-1804
}

\begin{abstract}
Smooth quantum hydrodynamic (QHD) model simulations of the resonant tunneling diode are presented which exhibit enhanced negative differential resistance (NDR) when compared to simulations using the original $O\left(\hbar^{2}\right)$ QHD model. At both $300 \mathrm{~K}$ and $77 \mathrm{~K}$, the smooth QHD simulations predict significant NDR even when the original QHD model simulations predict no NDR.
\end{abstract}

Keywords: Quantum hydrodynamic model, resonant tunneling diode

The original $O\left(\hbar^{2}\right)$ quantum hydrodynamic (QHD) equations have been remarkably successful in simulating the effects of electron tunneling through potential barriers including single and multiple regions of negative differential resistance and hysteresis in the current-voltage curves of resonant tunneling diodes. However, the model relies on an ad hoc replacement of derivatives of the potential with derivatives of the logarithm of the electron density in order to avoid infinite derivatives at heterojunctions.

Refs. [1] and [2] present an extension of the QHD model that is mathematically rigorous for classical potentials with discontinuities-as are present at heterojunction barriers in quantumsemiconductor devices. The stress tensor in this "smooth" QHD model actually cancels the leading singularity in the classical potential at a barrier and leaves a residual smooth effective potential with a lower potential height in the barrier region (see Fig. 1).

The smooth QHD equations have the same form as the classical hydrodynamic equations:

$$
\begin{gathered}
\frac{\partial n}{\partial t}+\frac{\partial}{\partial x_{i}}\left(n u_{i}\right)=0 \\
\frac{\partial}{\partial t}\left(m n u_{j}\right)+\frac{\partial}{\partial x_{i}}\left(m n u_{i} u_{j}-P_{i j}\right)=-n \frac{\partial V}{\partial x_{j}}-\frac{m n u_{j}}{\tau_{p}} \\
\frac{\partial W}{\partial t}+\frac{\partial}{\partial x_{i}}\left(u_{i} W-u_{j} P_{i j}+q_{i}\right) \\
=-n u_{i} \frac{\partial V}{\partial x_{i}}-\frac{\left(W-\frac{3}{2} n T_{0}\right)}{\tau_{w}}
\end{gathered}
$$

\footnotetext{
* The research of C.L.G. is supported in part by the U.S. Army Research Office under grant DAAH04-95-1-0122 and by the National Science Foundation under grant DMS-9706792. Corresponding author.

${ }^{\dagger}$ The research of C.R. is supported in part by the National Science Foundation under grant DMS-9706792.
} 


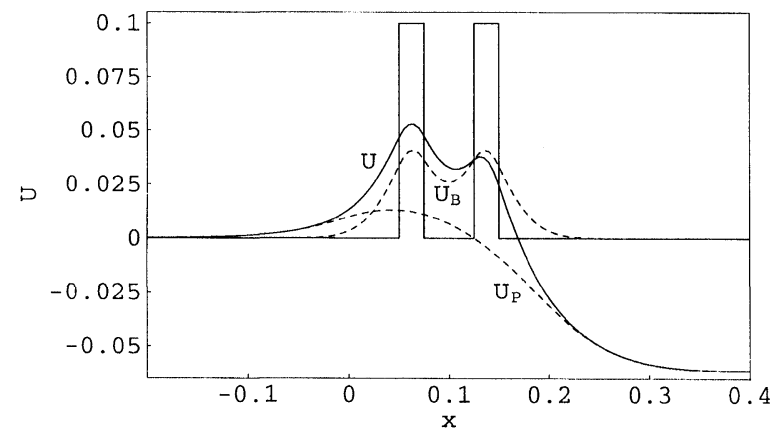

FIGURE 1 Smooth effective potentials $U, U_{B}$, and $U_{P}$ for $0.1 \mathrm{eV}$ double barriers at $300 \mathrm{~K} . x$ is in $\AA$

where repeated indices are summed over and where $n$ is the electron density, $\boldsymbol{u}$ is the velocity, $m$ is the electron mass, $P_{i j}$ is the stress tensor, $V$ is the classical potential energy, $W$ is the energy density, $\boldsymbol{q}$ is the heat flux, and $T_{0}$ is the ambient temperature. Collision effects are modeled by the relaxation time approximation, with momentum and energy relaxation times $\tau_{p}$ and $\tau_{w}$. These transport equations are coupled to Poisson's equation for the electric potential.

Quantum effects enter through the expressions for the stress tensor and the energy density:

$$
\begin{gathered}
P_{i j}=-n T \delta_{i j}-\frac{\hbar^{2} n}{4 m T} \frac{\partial^{2} \bar{V}}{\partial x_{i} \partial x_{j}} \\
W=\frac{3}{2} n T+\frac{1}{2} m n u^{2}+\frac{\hbar^{2} n}{8 m T} \nabla^{2} \bar{V}
\end{gathered}
$$

where $T=1 / \beta$ is the electron temperature and the "quantum" potential $\bar{V}$ is

$$
\begin{aligned}
& \bar{V}(\beta, \boldsymbol{x})= \frac{1}{\beta} \int_{0}^{\beta} d \beta^{\prime}\left(\frac{\beta^{\prime}}{\beta}\right)^{2} \\
& \int d^{3} X^{\prime}\left(\frac{2 m \beta}{\pi\left(\beta-\beta^{\prime}\right)\left(\beta+\beta^{\prime}\right) \hbar^{2}}\right)^{3 / 2} \\
& \times \exp \left\{-\frac{2 m \beta}{\left(\beta-\beta^{\prime}\right)\left(\beta+\beta^{\prime}\right) \hbar^{2}}\left(\boldsymbol{X}^{\prime}-\boldsymbol{x}\right)^{2}\right\} V\left(\boldsymbol{X}^{\prime}\right) .
\end{aligned}
$$

The quantum correction to the classical stress tensor and energy density is valid to all orders of $\hbar^{2}$ and to first order in $\beta V$, and involves both a smoothing integration of the classical potential over space and an averaging integration over temperature. The barrier height $\mathcal{B}$ is incorporated into the QHD transport equations (1)-(3) by replacing $V \rightarrow V+\mathcal{B}$. (Poisson's equation is not changed).

We define the 1D smooth effective potential in the momentum conservation equation (2) as the most singular part of $V-P_{11}$ :

$$
U=V+\frac{\hbar^{2}}{4 m T} \frac{d^{2} \bar{V}}{d x^{2}}
$$

The double integration over both space and inverse temperature provides sufficient smoothing so that the $P_{11}$ term in the smooth effective potential cancels the leading singularity in the classical potential at a barrier.

The 1D steady-state smooth QHD equations are discretized [3] using a conservative upwind method adapted from computational fluid dynamics. The discretized equations are then solved by a damped Newton method.

There are two contributions to the quantum potential $\bar{V}$ : the double barrier potential and the "self-consistent" electric potential from Poisson's equation. Note that second derivatives of $\bar{V}$ appear in the stress tensor and energy density, which then are differenced in the smooth QHD transport equations. Thus we compute

$$
\bar{V}^{\prime \prime}=\bar{V}_{B}^{\prime \prime}+\bar{V}_{P}^{\prime \prime}
$$

$\bar{V}_{B}^{\prime \prime}$ is just computed once since it only depends on the barriers and not on the applied voltage or state variables $\left(n, u, T, V_{P}\right)$. In computing $\bar{V}_{P}^{\prime \prime}$, we first use Poisson's equation to obtain

$$
\begin{aligned}
\bar{V}_{P}^{\prime \prime}(\beta, x)= & \frac{e^{2}}{\varepsilon} \int_{0}^{\beta} \frac{d \beta^{\prime}}{\beta}\left(\frac{\beta^{\prime}}{\beta}\right)^{2} \\
& \int d X^{\prime}\left(\frac{2 m \beta}{\pi\left(\beta-\beta^{\prime}\right)\left(\beta+\beta^{\prime}\right) \hbar^{2}}\right)^{3 / 2}
\end{aligned}
$$




$$
\begin{aligned}
& \times \exp \left\{-\frac{2 m \beta}{\left(\beta-\beta^{\prime}\right)\left(\beta+\beta^{\prime}\right) \hbar^{2}} X^{\prime 2}\right\} \\
& \left(N_{D}\left(X^{\prime}+x\right)-n\left(X^{\prime}+x\right)\right)
\end{aligned}
$$

where $N_{D}$ is the density of donor ions. Then to efficiently compute the convolution (9), we take advantage of properties of the Fourier transform.

We present simulations of a GaAs resonant tunneling diode with $\mathrm{Al}_{x} \mathrm{Ga}_{1-x} \mathrm{As}$ double barriers at $300 \mathrm{~K}(77 \mathrm{~K})$. The barrier height $\mathcal{B}$ is set equal to $0.1(0.05) \mathrm{eV}$. The diode consists of $n^{+}$source (at the left) and drain (at the right) regions with the doping density $N_{D}=10^{18} \mathrm{~cm}^{-3}$, and an $n$ channel with $N_{D}=5 \times 10^{15} \mathrm{~cm}^{-3}$. The channel is 200 (250) $\AA$ long, the barriers are 25 (50) $\AA$ wide, and the quantum well between the barriers is $50 \AA$ wide. Note that the device has $50 \AA$ spacers between the barriers and the contacts. We have chosen parameters to highlight differences between the original and smooth QHD models.

Figure 1 illustrates the smooth effective potentials $U_{B}$ (for the barriers), $U_{P}$ (the Poisson contribution), and $U$ (barrier plus Poisson contributions) for the resonant tunneling diode at $300 \mathrm{~K}$ at the voltage $V=0.056$ where the $I-V$ curve peaks in Figure 2.

Smooth QHD simulations of the resonant tunneling diode exhibit enhanced negative differential resistance when compared to simulations

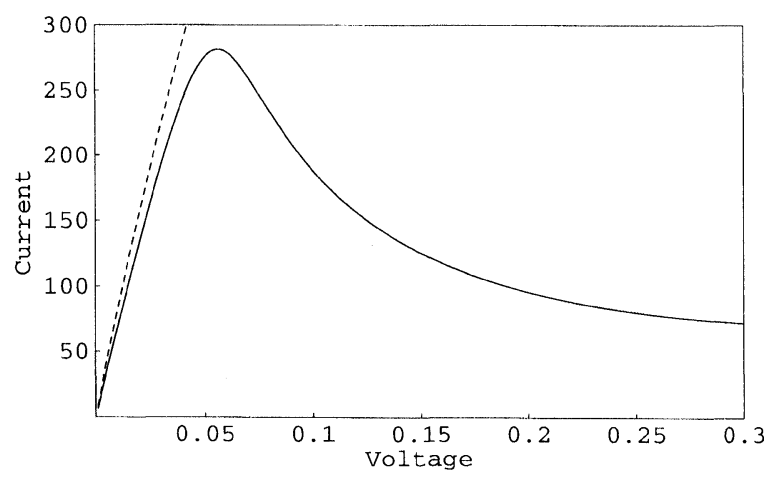

FIGURE 2 Current density in kiloamps $/ \mathrm{cm}^{2}$ vs. voltage for the resonant tunneling diode at $300 \mathrm{~K}$. The solid curve is the smooth QHD computation and the dotted line is the $O\left(\hbar^{2}\right)$ computation. The barrier height is $0.1 \mathrm{eV}$. using the original $O\left(\hbar^{2}\right)$ QHD model. The currentvoltage curve for the resonant tunneling diode at $300 \mathrm{~K}$ is plotted in Figure 2 and at $77 \mathrm{~K}$ is plotted in Figure 3. It is interesting that the original $O\left(\hbar^{2}\right)$ QHD model (see Refs. [4, 3] and references therein) predict very different $I-V$ curves - in fact, at both $300 \mathrm{~K}$ and $77 \mathrm{~K}$ the original $O\left(\hbar^{2}\right)$ QHD model fails to produce negative differential resistance for these devices.

Simulations of the resonant tunneling diode using the Wigner-Boltzmann/Poisson equations are planned to determine which of the QHD models gives better agreement with the more complete quantum kinetics. In these comparisons, we will use Fokker-Planck collision terms with a relaxation time $\tau$ in the Wigner-Boltzmann equation, which then implies momentum and energy relaxation times $\tau_{p}=\tau$ and $\tau_{w}=\tau / 2$ in the QHD models.

We will then be able to answer the question: In what parameter range and how accurately do the smooth QHD solutions (first three moments) reflect the solutions to the full Wigner-Boltzmann equation? There should be a technologically important range of parameters (device size, ambient temperature, potential barrier height, applied voltage, semiconductor material, etc.) in which the smooth QHD model gives solutions and $I-V$ curves that are very close to those given by the full WignerBoltzmann/Poisson system.

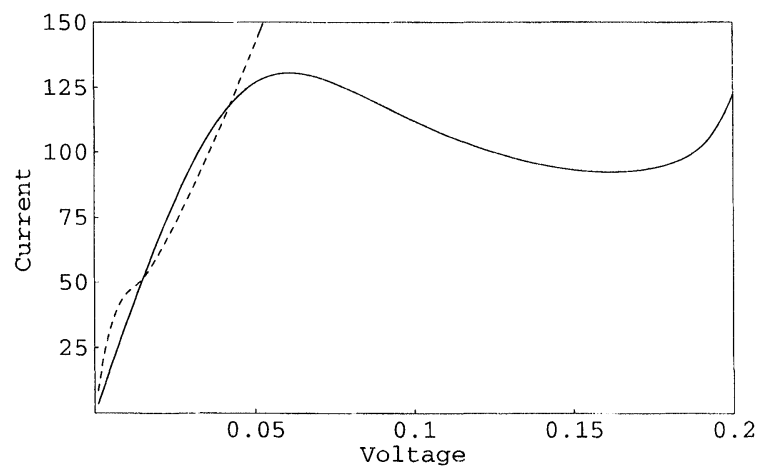

FIGURE 3 Current density in kiloamps $/ \mathrm{cm}^{2}$ vs. voltage for the resonant tunneling diode at $77 \mathrm{~K}$. The solid curve is the smooth QHD computation and the dotted line is the $O\left(\hbar^{2}\right)$ computation. The barrier height is $0.05 \mathrm{eV}$. 


\section{References}

[1] Gardner, C. L. and Ringhofer, C. (1996). "Smooth quantum potential for the hydrodynamic model," Physical Review, E 53, pp.157-167.

[2] Gardner, C. L. and Ringhofer, C. (1998). "Approximation of thermal equilibrium for quantum gases with discontinuous potentials and application to semiconductor devices," SIAM Journal on Applied Mathematics, accepted for publication.

[3] Gardner, C. L. (1994). "The quantum hydrodynamic model for semiconductor devices", SIAM Journal on Applied Mathematics, 54, pp. 409-427.

[4] Grubin, H. L. and Kreskovsky, H. L. (1989). "Quantum moment balance equations and resonant tunnelling structures", Solid-State Electronics, 32, pp. 1071-1075.

\section{Authors' Bigraphies}

Carl L. Gardner is Professor of Mathematics at Arizona State University. His current research interests lie in classical and quantum semiconductor device simulation and computational fluid dynamics.

Christian Ringhofer is Professor of Mathematics at Arizona State University. His current research interests include classical and quantum transport equations and moment models for semiconductor device modeling. 

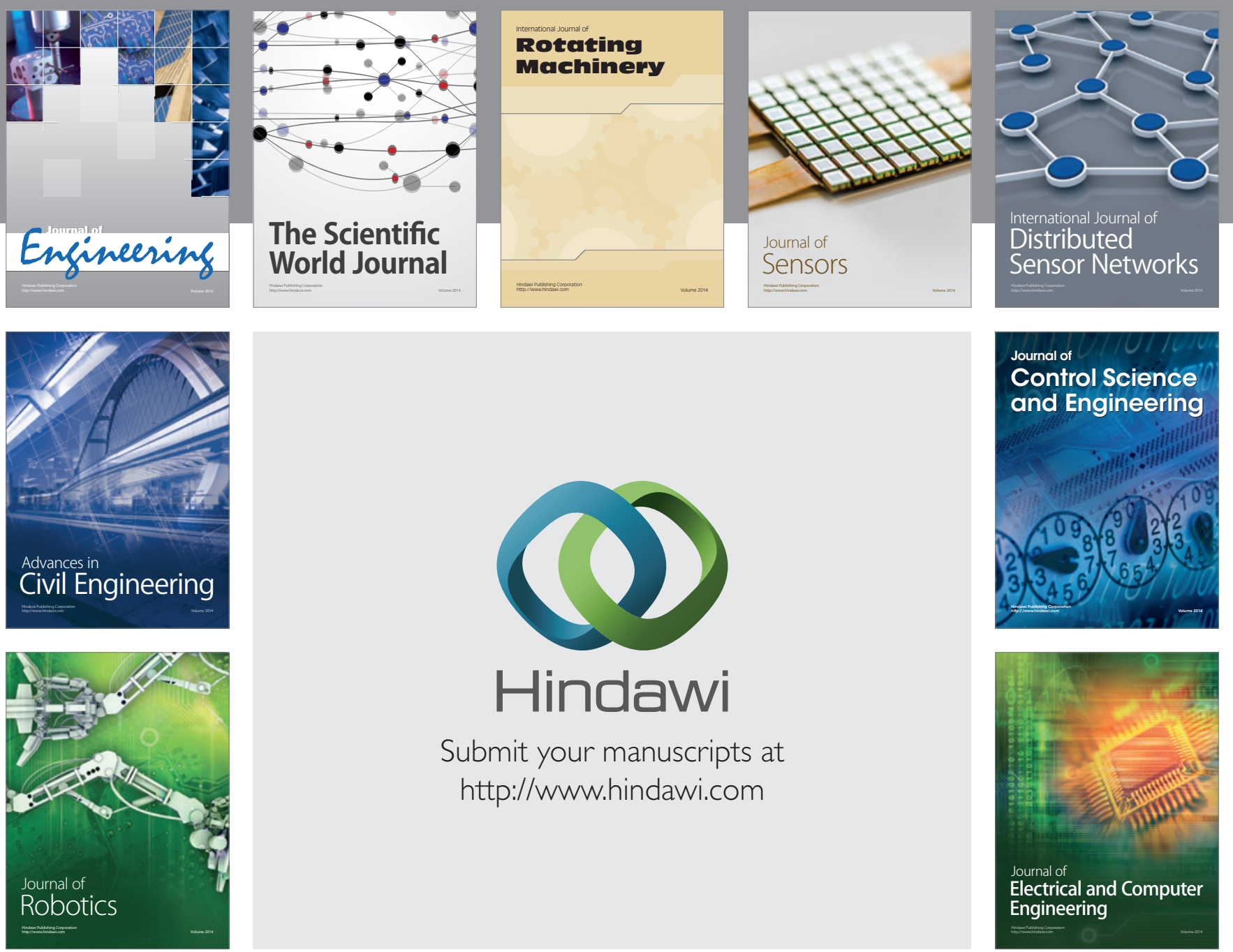

Submit your manuscripts at

http://www.hindawi.com
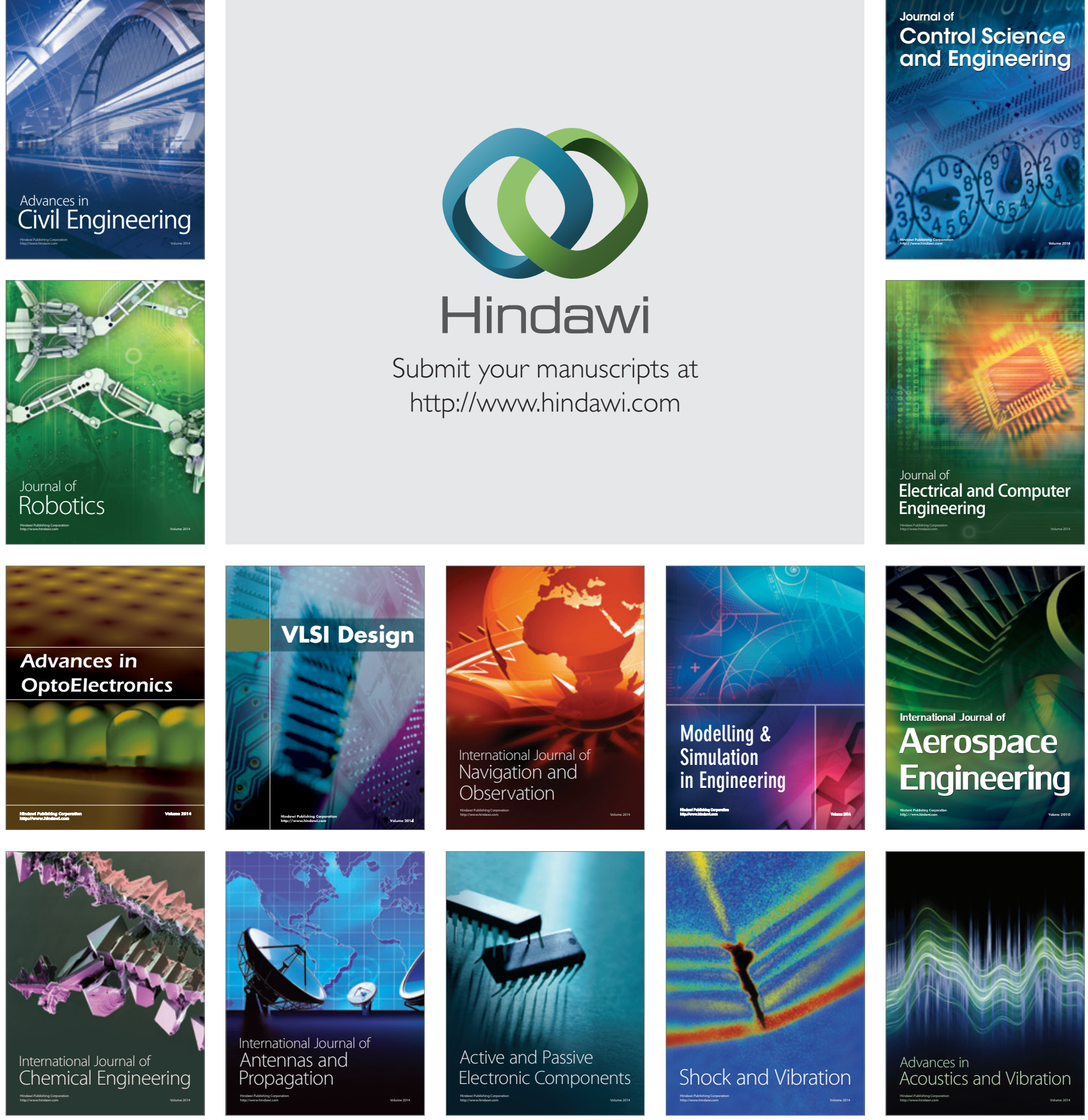